\title{
Learning to Improve Capture Steps for Disturbance Rejection in Humanoid Soccer
}

\author{
Marcell Missura, Cedrick Münstermann, Philipp Allgeuer, Max Schwarz, \\ Julio Pastrana, Sebastian Schueller, Michael Schreiber, and Sven Behnke \\ Autonomous Intelligent Systems, Computer Science, Univ. of Bonn, Germany \\ \{missura, schreiber\}@ais.uni-bonn.de, behnke@cs.uni-bonn.de \\ http://ais.uni-bonn.de
}

\begin{abstract}
Over the past few years, soccer-playing humanoid robots have advanced significantly. Elementary skills, such as bipedal walking, visual perception, and collision avoidance have matured enough to allow for dynamic and exciting games. When two robots are fighting for the ball, they frequently push each other and balance recovery becomes crucial. In this paper, we report on insights we gained from systematic push experiments performed on a bipedal model and outline an online learning method we used to improve its push-recovery capabilities. In addition, we describe how the localization ambiguity introduced by the uniform goal color was resolved and report on the results of the RoboCup 2013 competition.
\end{abstract}

\section{Introduction}

In the RoboCup Humanoid League, robots with a human-like body plan compete against each other in soccer games. The robots are largely self-constructed, and are divided into three size classes: KidSize $(<60 \mathrm{~cm})$, TeenSize $(90-120 \mathrm{~cm})$, and AdultSize $(>130 \mathrm{~cm})$. The TeenSize robots started to play 2 vs. 2 soccer games in 2010 and moved to a larger soccer field of $9 \times 6 \mathrm{~m}$ in the year 2011 . In addition
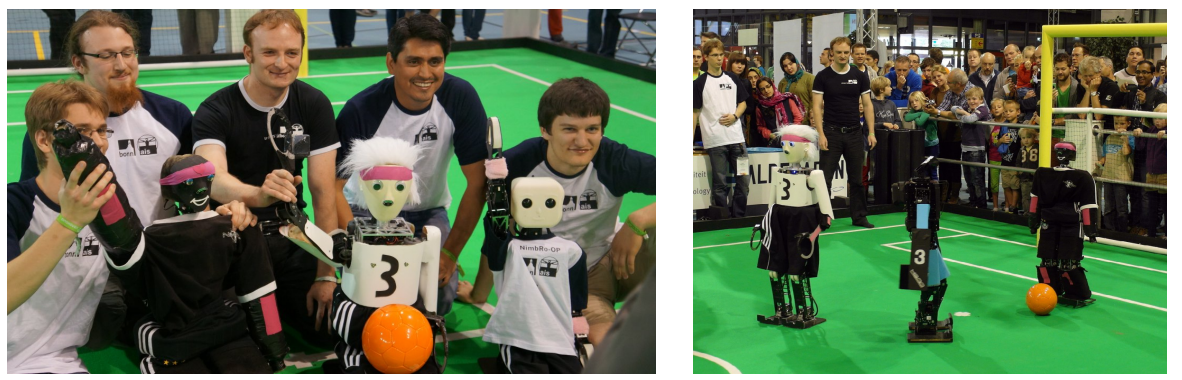

Fig. 1. Left: Team NimbRo with robots Dynaped, Copedo, and NimbRo-OP. Right: Team NimbRo vs. CIT-Brains in the RoboCup 2013 finals. 
to the soccer games, the robots face technical challenges, such as throwing the ball into the field from a side line.

For RoboCup 2013, the color coding of the goal posts was unified to yellow for both goals and the landmark poles at the ends of the center line were removed. Consequently, it was not possible anymore to determine the unambiguous position of a robot on the field based only on visual cues, which constitutes a problem for localization. However, most teams were able to implement suitable solutions and were able to reliably drive the ball towards the opponent goal. Our approach to disambiguate localization was to integrate a compass as an additional source of information. More details are given in Section 3 .

Inspired by the success of the DARwIn-OP robot, we have constructed a TeenSize open platform, the NimbRo-OP. Following the same spirit, the NimbRo-OP is a low-cost robot that is easy to construct, maintain, and extend. It is intended to provide access to a humanoid robot platform for research. The NimbRo-OP has matured enough to participate in the competitions. It participated in the Technical Challenges and scored its first official competition goal in the main event. More information about the NimbRo-OP is given in Section 5 .

Bipedal walking is a crucial skill in robot soccer. It determines the success of a team to a substantial degree. Humanoid robots must be able to walk up to a ball and kick it, preferably without losing balance and falling to the ground. While most of the teams have mastered the skill of unperturbed walking on flat terrain, solutions to recover from strong disturbances, such as collisions with opponents, are not yet widespread. In ongoing research, team NimbRo has developed a stable bipedal gait control framework that has been designed to absorb strong perturbations. In Section 6, we report on the insights we gained from systematic push experiments, and introduce an online learning method that we used to improve push recovery capabilities. The learning controller is able to adjust the step size and recover balance quicker than the underlying simplified mathematical model.

\section{Mechatronic Design of NimbRo TeenSize Robots}

The mechatronic design of our robots is focused on robustness, weight reduction, and simplicity. All our robots are constructed from milled carbon fiber and aluminum parts that are assembled to rectangular shaped legs and flat arms. We use Dynamixel EX-106 and EX-106+ servos for the actuation of our classic robots Dynaped and Copedo. These robots are also equipped with spring-loaded protective joints that yield to mechanical stress and can snap back into place automatically. More information about the mechanical structure of the NimbRo classic robots can be found in 1] and 2. The NimbRo-OP robot has a slightly different design with a reduced complexity. It is equipped with 6 DOF legs and 3 DOF arms that offer enough flexibility to walk, to kick, and to get up from the floor after falling. It is actuated by servos from the Dynamixel MX series. The mechatronic structure of the NimbRo-OP is best described in [3]. 


\section{Perception}

For visual perception of the game situation, we detect the ball, goal-posts, penalty markers, field lines, corners, T-junctions, X-crossings, obstacles, team mates, and opponents utilizing color, size and shape information. We estimate distance and angle to each detected object by removing radial lens distortion and by inverting the projective mapping from field to image plane.

For proprioception, we use the joint angle feedback of the servos and apply it to the kinematic robot model using forward kinematics. Before extracting the location and the velocity of the center of mass, we rotate the kinematic model around the current support foot such that the attitude of the trunk matches the angle we measured with the IMU. Temperatures and voltages are also monitored for notification of overheating or low batteries.

For localization, we track a three-dimensional robot pose $(x, y, \theta)$ on the field using a particle filter 4. The particles are updated using a linear motion model. Its parameters are learned from motion capture data [5]. The weights of the particles are updated according to a probabilistic model of landmark observations (distance and angle) that accounts for measurement noise. To handle unknown data association of ambiguous landmarks, we sample the data association on a per-particle basis. The association of field line corner and T-junction observations is simplified using the orientation of these landmarks. Further details can be found in [6] and [2].

Integration of a compass: This year, we extended our sensory systems with a compass in order to help the particle filter to disambiguate the localization on the field. As starting from 2013 both goals have the same color and there are no landmarks that allow unambiguous localization based only on visual cues, it was necessary to add an additional source of information other than the objects detected by the computer vision. Using the compass output as observation of the global orientation in the particle filter greatly helps to reduce the number of hypothesis that can accumulate in the particle distribution. Figure 2 shows such an example. The robot observes a situation in the corner of the field, where field lines, L-shaped line crossings and a goal post have been successfully detected. Despite the high number of observations that the particles can be weighted with, two equally valid hypotheses form, as shown by the particle distribution
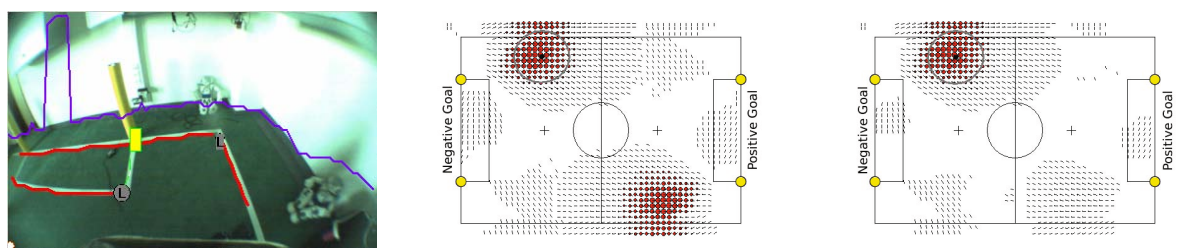

Fig. 2. Effect of the compass on localization confidence. The observed scene in the camera image (left) leads to two hypothesis peaks in the particle distribution of the particle filter (center). Adding the compass reading as an additional observation disambiguates the position estimation (right). 
in the center. Adding the global heading as additional observation reduces the probability of particles that are facing in a wrong direction. Thereby one of the hypothesis in this example is invalidated (right). As an additional benefit of using a compass, we found that it not only improves localization, but also the effectiveness of our soccer behaviors. This is due to the fact that the rough direction of the opponent goal is always known. Thus, the ball is always moved in the right direction, even in cases where the particle filter reports a wrong pose.

\section{Behavior Control}

We control our robots using a layered framework that supports a hierarchy of reactive behaviors [7. When moving up the hierarchy, the update frequency of sensors, behaviors, and actuators decreases, while the level of abstraction increases. Currently, our implementation consists of three layers. The lowest, fastest layer is responsible for generating motions, such as walking [8] —including capture steps [9], kicking, get-up motions [10, and the goalie dive [11. At the next higher layer, we model the robot as a simple holonomic point mass that is controlled with the force field method to generate ball approach trajectories, ball dribbling sequences, and to implement obstacle avoidance. The topmost layer of our framework takes care of team behavior, game tactics and the implementation of the game states as commanded by the referee box. Please refer to [2] for further details.

\section{$5 \quad$ NimbRo-OP TeenSize Robot}

Our main innovation this year was the development of the NimbRo-OP robot along with a ROS framework based robot soccer software. The software contains many modules for basic functions required for playing soccer that we either started from scratch, or ported from our classic NimbRo system. In the now second release [12, the software package contains a compliant servo actuation module [13] and a visual motion editing component. Motions are replayed with a non-linear keyframe interpolation technique that allows to generate smooth and continuous motions while respecting configurable acceleration and velocity bounds. Kicking and get-up motions have been successfully implemented. For walking, we use a port of the same gait generator that we use for

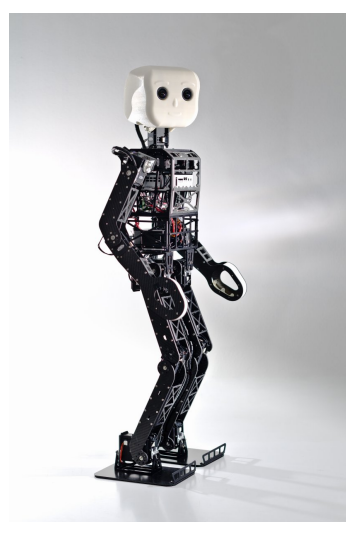

Fig. 3. The NimbRo-OP our classic robots [8]. For higher-level behavior control, we ported the NimbRo hierarchical reactive behavior architecture [7] [14] and the implementations of simple soccer behaviors within, such as searching for the ball, walking up to the ball and dribbling the ball. The vision processing module was rewritten from 
scratch as a ROS module along with accompanying tools for camera and color calibration. Utilizing a camera with higher resolution and more available processing power, we improved the quality of our object detection, which is described in [12] in more detail. A particle filter-based localization module is also provided. Apart from the core soccer software itself, graphical software components are available to maintain configuration parameters and to log the state of the system in great detail to support debugging and monitoring during games.

\section{Online Learning of Lateral Balance}

In recent years, team NimbRo has developed a gait control framework capable of recovering from pushes that are strong enough to force a bipedal walker to adjust step-timing and foot-placement. Only lateral balance mechanisms [9] have been used in competitions so far, but in simulation, the framework is now able to absorb pushes from any direction at any time during the gait cycle [15]. In a nutshell, the Capture Step Framework is based on an extremely simplified state representation in the form of a point mass that is assumed to behave like a linear inverted pendulum. A decomposition of the lateral and sagittal dimensions into independent entities, and a sequential computation of step-timing, zeromoment point and foot-placement control parameters facilitates the closed-form mathematical expression of our balance controller. Modeling, however, can only take one so far. Complex full-body dynamics, sensor noise, latency, imprecise actuation, and simplifying modeling assumptions will always result in errors that can limit the balancing capabilities of a humanoid robot. A good way to increase the efficiency of a model based approach are online learning techniques that can measure performance during walking and adjust the output of modelbased push-recovery strategies.

Focusing on the simplified purely lateral setting, we have successfully implemented an online learning algorithm that learns the foot-placement error during disturbed walking on the spot and subtracts it from the model output in order to improve push recovery capabilities. In the following section, we briefly outline the concepts of lateral balance and introduce our evaluation method that can quantify and visualize the effects of isolated balance components. Subsequently, we describe the online learning algorithm we used, and show experimental results to verify the achieved improvement.

\subsection{Lateral Gait Control}

The pendulum-like dynamics of human walking has been long known to be a principle of energy-efficient locomotion [16]. Figure 4 shows stick diagrams of the idealized sagittal and lateral pendulum motions projected on the sagittal plane and the frontal plane. Interestingly, the sagittal and lateral motions exhibit strongly distinct behaviors. In the sagittal plane, the center of mass vaults over the pivot point in every gait cycle, while in the frontal plane, the center of mass oscillates between the support feet and never crosses the pendulum pivot point. 

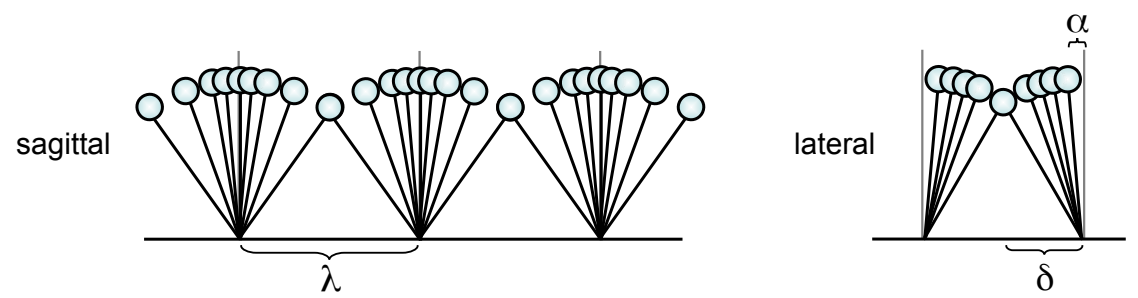

Fig. 4. Stick diagrams of idealized pendulum-like sagittal and lateral motion of a compass gait. In sagittal direction, the center of mass crosses the pendulum pivot point in every gait cycle, while in lateral direction it oscillates between the support feet. Parameter $\lambda$ defines the stride length in the sagittal direction, parameter $\alpha$ denotes the characteristic lateral apex distance, and $\delta$ defines the support exchange location in the center of the step.

It is crucial not to tip over sideways, as the recovery from such an unstable state requires challenging motions that humanoid robots have difficulties performing.

The perpetual lateral oscillation of the center of mass appears to be the primary determinant of step timing. Disobeying the right timing can quickly destabilize the system after a disturbance, even if the disturbance itself would not have directly resulted in a fall [17. Furthermore, we can identify two characteristic parameters in the lateral direction. We denote the minimal distance between the pivot point and the center of mass and that occurs at the apex of the step as $\alpha$. The apex distance provides a certain margin for error. While during undisturbed walking the apex distance stays near $\alpha$ in every step, a push in the lateral direction can result in a smaller apex distance. As long as the apex distance is greater than zero, the center of mass will return and the walker will not tip over the support foot. Sooner or later, returning center of mass trajectories are guaranteed to reach the support exchange location that we denote as $\delta$. While the support exchange location varies with increasing lateral walking velocity, for now we limit our setting to walking on the spot with zero velocity of locomotion and therefore we can assume $\delta$ to be a constant as well. To identify the model parameters $\alpha$ and $\delta$ for a real or a simulated biped, we induce the lateral oscillation by generating periodic, open-loop step motions using the walk algorithm described in [8]. Then, $\alpha$ and $\delta$ can be found by averaging the measured center of mass locations at the step apex and in the moment of the support exchange.

As a consequence of the principles described above, we can formulate the following control laws for our balance control computations:

- The timing of the step is determined by the moment when the center of mass reaches the nominal support exchange location $\delta$.

- The lateral step size is chosen so that the center of mass will pass the following step apex with a nominal distance $\alpha$ with respect to the pivot point.

Formally, our balance controller is a function

$$
(T, F)=\mathcal{B}(y, \dot{y})
$$


that computes the step time $T$ and the footstep location $F$ as a function of the current state of the center of mass $(y, \dot{y})$. Here, $y$ denotes the location of the center of mass along the lateral axis with respect to a right hand coordinate frame placed on the support foot, and $\dot{y}$ is the velocity of the center of mass. The step time $T$ and the footstep location $F$ are passed on to a motion generator that generates stepping motions with an appropriate frequency and leg swing amplitude. For the understanding of the experiments performed in this work, a conceptual insight of the lateral control laws presented above is sufficient. For more detailed information, we refer the reader to [15].

\subsection{Experimental Setup}

Using a physical simulation software, we performed a series of systematic push experiments on a simulated humanoid robot with a total body weight of $13.5 \mathrm{~kg}$ and a roughly human-like mass distribution. While the robot is walking on the spot, it is pushed in the lateral direction with an impulse targeted at the center of mass. After the impulse, the robot has some time to recover, before the next impulse is generated. If the robot falls, it is reset to a standing position and it is commanded to start walking again. The magnitude of the impulse is randomly sampled from the range $[-9.0,9.0] \mathrm{Ns}$, where the sign of the impulse determines its direction (left or right). We generate 400 pushes for each of four balance controllers of increasing complexity:

- No Feedback: The controller ignores the pushes and does nothing. The robot executes an open-loop gait with a fixed frequency and step size.

- Timing: The controller adjusts only the timing of the step, but not the footstep location.

- Timing + Step Size: The controller adjusts the timing and the size of the steps using the mathematical model.

- Timing + Step Size + Learning: The controller responds to the disturbances using not only the model-based computation of the timing and the step size, but also a learned error that we subtract from the predicted step size. The error is learned online during the experiment.

The input space we use for learning is the lateral state space $\mathcal{S}=[y, \dot{y}] \in \mathbb{R}^{2}$ of the center of mass. When the support foot is the left foot, we flip the signs of $y$ and $\dot{y}$ in order to exploit symmetry. During the experiment, the robot measures the efficiency of its steps and estimates an error that expresses a gradient, i.e. a desired scalar increase or decrease in the step size. The error is measured when the center of mass is at the step apex. It is given as simply the deviation from the nominal apex distance $\alpha$. From the inverted pendulum model it follows intuitively that if the apex distance is greater than $\alpha$, the step size was too large, and if the apex distance is smaller than $\alpha$, the step size was too small. At the end of the step, we update the value of a function approximator for each of the states $(y, \dot{y})_{i \in I}$ that were encountered during the step. The update rule is

$$
f\left((y, \dot{y})_{i}\right)=f\left((y, \dot{y})_{i}\right)+\eta\left(y_{i_{a}}-\alpha\right), i \in I,
$$




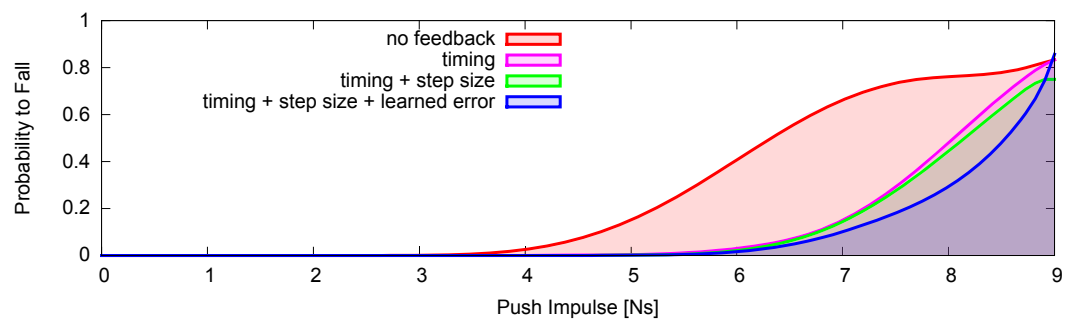

Fig. 5. Probability to fall versus the magnitude of the push impulse for four different controllers of increasing complexity

where $f\left((y, \dot{y})_{i}\right)$ is the value of the function approximator for the state $(y, \dot{y})_{i}, y_{i_{a}}$ is the center of mass location that was measured at the step apex, and $\eta=0.2$ is the learning rate. The function approximator is initialized with a value of 0 before learning. The step parameters that are passed on to the step motion generator are then

$$
(T, F)=\mathcal{B}(y, \dot{y})-(0, f(y, \dot{y}))
$$

\subsection{Evaluation of Results}

Using the data we collected during the experiments, we can compare the efficiency of the four controllers. Figure 5 shows the probability to fall against the magnitude of the impulse and gives an impression of the push resistance of the controllers. Interestingly, the open-loop walk alone is able to handle pushes up to a strength of $3 \mathrm{Ns}$, in such a case returning slowly to a limit cycle. However, the three feedback controllers clearly increase the minimum impact required to make the robot fall and improve the ability to absorb an impact over the entire range of impulse strengths. The results of the three feedback controllers do not differ from each other significantly, leading to the conclusion that using the right step timing is already sufficient to predominantly stabilize returning center of mass trajectories. Why this effect can be achieved with step timing alone has a reasonable explanation. When the robot receives a push from the side, it typically first tilts towards the support leg and the center of mass approaches the outer edge of the support foot. If the robot was pushed in the direction away from the support leg, it will automatically tip onto the other leg in the center of the step, which leads to the same situation. Now, when the center of mass is moving towards the outer edge of the support foot, the robot may shorten the support leg if it does not adjust the motion timing, as internally the support leg is thought to be the swing leg at that time. This accelerates the center of mass additionally towards the support leg and reduces the lever arm, helping the robot to tip over the outer edge of the foot. Furthermore, the robot is likely to touch the floor with the other foot and can further accelerate itself in the wrong direction. And finally, if the center of mass returns, and it is moving away from the support leg, a badly timed extension of the support leg just before the support exchange adds energy to the lateral motion and increases the probability 

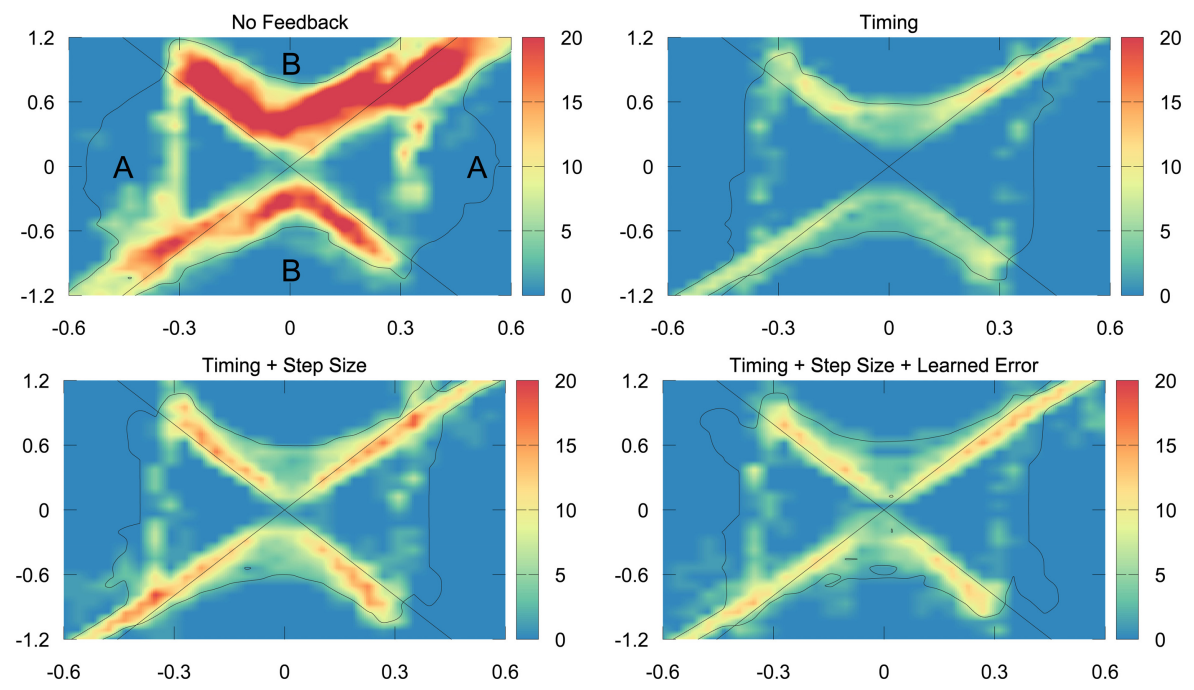

Fig. 6. Heat maps of unstable regions of the lateral phase space. Color coding marks the areas that have been crossed by falling trajectories. Thin black lines contour the cells that were visited at least ten times during the experiments. Straight zero-energy lines partition the phase space into stable regions of negative orbital energy (A), and unstable regions of positive orbital energy (B).

to tip over on the other side. Using adaptive timing, all of these undesired effects vanish. The adaptation of step timing prevents the robot from destabilizing itself due to badly timed leg motions in oblique poses and maximizes the minimal tip-over impulse to the value that can be passively absorbed. Using the torso as a reaction mass for active balancing could further increase the minimal tip-over impulse, but this is not in our scope at this time.

For a closer look, Figure 6] shows heat maps of the lateral phase space that were generated by backtracking from every fall to the first frame of a push and incrementing each grid cell that was touched by the center of mass on the way. The values of the cells are then used for color coding the unstable regions of the phase space for each controller. The thin black contours bound the regions of cells that were visited at least ten times during the experiments. The straight zero-energy lines are computed from the linear inverted pendulum model that is used to drive the feedback loops. The zero-energy lines partition the phase space into regions that we would expect to find based on model assumptions. The areas marked with the letter 'A' are regions of negative orbital energy. This is where all returning center of mass trajectories are located and stable lateral oscillations can take place. The sectors marked with the letter 'B' are of positive orbital energy and contain state trajectories that will inevitably cross the pivot point and tip over. The model is reflected by the experimental data, as the vast majority of the states encountered between a push and a fall are located in the unstable areas of the heat maps. The fall trajectories of all controllers must 

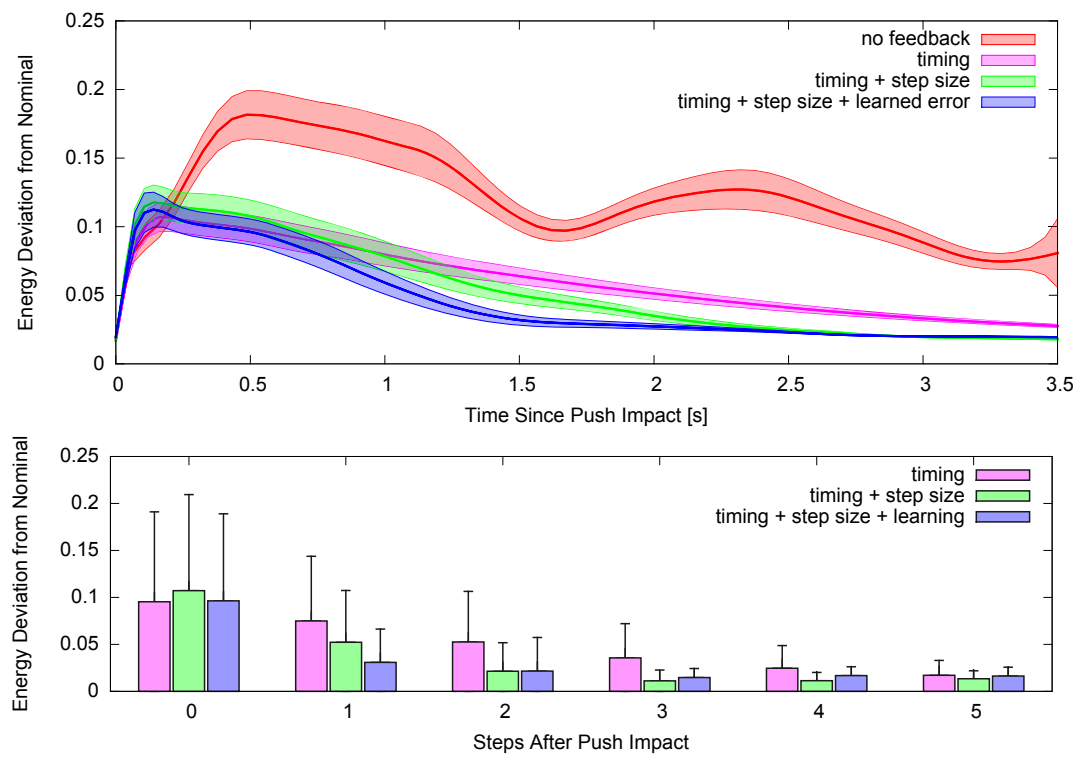

Fig. 7. Development of the lateral orbital energy after a push synchronized at the push impact (top), and at the individual steps after the push (bottom). While the "Timing" controller monotonically returns to a desired level of orbital energy, the adjustment of step size helps the robot to return to the nominal energy level much faster. The open-loop controller cannot be sensibly synchronized with the feedback controllers and thus it has been omitted from the bottom plot.

originate from the stable region, since the push is always applied in a stable state of the robot. The push changes the state trajectory abruptly and transfers it into the unstable section 'B'. It is evident that the heat map of the open-loop controller contains a much larger number of falls. The heat maps of the three feedback controllers look very similar with a strongly reduced number of falls in comparison with the "No Feedback" experiment. Again, we can conclude that step timing adaptation plays a pivotal role in preventing a fall.

In order to answer the question of how a bipedal walker can benefit from a well chosen step size, Figure 7 shows the development of the orbital energy after the disturbance in the cases where the robot did not fall. In the top half of the plot, the time series of the orbital energy deviation from a nominal value has been synchronized at the moment of the push impact. Since the open-loop controller has a tendency to amplify the push impulse, the peak energy shortly after the push is significantly higher. The wave-like form of the energy curve suggests that the open-loop controller occasionally disturbs itself. When using only timing feedback, the disturbance amplification and the self disturbances disappear and the orbital energy returns monotonically to a desired level. With the addition of a computed step size, the robot can absorb the orbital energy much faster. The controller with the learned step size error shows the best performance in 
terms of orbital energy dissipation. In the bottom half of the plot, the energy level with respect to the nominal value has been synchronized at the individual steps after the push. The fixed-frequency steps of the open-loop controller cannot be sensibly synchronized with the timed steps of the feedback controllers and thus have been omitted from the bottom plot. The first group of boxes show the energy deviation that has been measured during the step that was pushed. The second group of boxes at the index 1 represent the "capture step", the first step after the push. As in theory a full recovery is possible with one step, the efficiency of the capture step is of particular interest. The efficiency of a step can be computed as $1-\frac{e_{s}}{e_{s-1}}$, where $e_{s-1}$ and $e_{s}$ are the excess energy levels before and after the step. The step efficiency of the step timing controller is $21 \%$. Adding the step size modification improves the step efficiency to $51 \%$, and learning further increases the energy absorption rate to $68 \%$. Accelerating the return to a nominal, stable state has a positive effect on overall bipedal stability. The walker is ready to face the next disturbance in a shorter amount of time and thus not only the magnitude, but also the frequency of impulses that the robot can handle, is increased.

\section{Conclusions}

The TeenSize class experienced an uplift during the 2013 competition. Five teams were at the competition site and played games with more than one operational robot on the field from each team. Several technical challenges were completed. All teams were able to advance their software to cope with the new challenge of localization with symmetrical landmarks.

In the final, our robots met team CIT-Brains from Japan. In the beginning of the match, each team played with two players on the field. CIT-Brains played an offensive strategy with two strikers while team NimbRo designated one player as goal keeper. The CIT team managed to press onward towards the NimbRo goal, but the NimbRo robots defended against the attacks reliably. The obstacle avoidance feature of the CIT robots appeared to be a bit too aggressive and they approached the NimbRo robots too closely and often stepped on their toes, which made the CIT robots fall over. NimbRo striker Copedo used the opening gaps to score. Team NimbRo successfully demonstrated dynamic role assignment that temporarily assigned the goal keeper Dynaped the striker role when Copedo had to be taken out of the game. While in the second half, team CIT Brains had to reduce the number of players to one due to technical difficulties, team NimbRo managed to maintain two operational players throughout the game and scored reliably. Consequently, team NimbRo won the finals with a score of 4:0 and successfully defended its title for the fifth time in a row.

The stability of the gait of our robots and their robustness to disturbances was one of the key factors for our success. The online learning method outlined in this work will contribute to even faster stabilization of bipedal walking in future competitions. 
Acknowledgment. This work is supported by Deutsche Forschungsgemeinschaft (German Research Foundation, DFG) under grants BE 2556/6 and BE 2556/10.

\section{References}

1. Missura, M., Münstermann, C., Mauelshagen, M., Schreiber, M., Behnke, S.: RoboCup 2012 Best Humanoid Award Winner NimbRo TeenSize. In: Chen, X., Stone, P., Sucar, L.E., van der Zant, T. (eds.) RoboCup 2012. LNCS (LNAI), vol. 7500, pp. 89-93. Springer, Heidelberg (2013)

2. Lee, D.D., et al.: RoboCup 2011 Humanoid League winners. In: Röfer, T., Mayer, N.M., Savage, J., Saranlı, U. (eds.) RoboCup 2011. LNCS, vol. 7416, pp. 37-50. Springer, Heidelberg (2012)

3. Schwarz, M., Schreiber, M., Schueller, S., Missura, M., Behnke, S.: NimbRo-OP Humanoid TeenSize Open Platform. In: Proceedings of 7th Workshop on Humanoid Soccer Robots, IEEE Int. Conf. on Humanoid Robots, Osaka, Japan (2012)

4. Thrun, S., Burgard, W., Fox, D.: Probabilistic Robotics. MIT Press (2001)

5. Schmitz, A., Missura, M., Behnke, S.: Learning footstep prediction from motion capture. In: Ruiz-del-Solar, J., Chown, E., Plöger, P.G. (eds.) RoboCup 2010. LNCS (LNAI), vol. 6556, pp. 97-108. Springer, Heidelberg (2010)

6. Schulz, H., Behnke, S.: Utilizing the structure of field lines for efficient soccer robot localization. Advanced Robotics 26, 1603-1621 (2012)

7. Behnke, S., Stückler, J.: Hierarchical reactive control for humanoid soccer robots. Int. Journal of Humanoid Robots (IJHR) 5, 375-396 (2008)

8. Missura, M., Behnke, S.: Self-stable Omnidirectional Walking with Compliant Joints. In: Proceedings of 8th Workshop on Humanoid Soccer Robots, IEEE Int. Conf. on Humanoid Robots, Atlanta, USA (2013)

9. Missura, M., Behnke, S.: Lateral capture steps for bipedal walking. In: Proceedings of IEEE Int. Conf. on Humanoid Robots (Humanoids) (2011)

10. Stückler, J., Schwenk, J., Behnke, S.: Getting back on two feet: Reliable standing-up routines for a humanoid robot. In: Proceedings of The 9th Int. Conf. on Intelligent Autonomous Systems, IAS-9 (2006)

11. Missura, M., Wilken, T., Behnke, S.: Designing effective humanoid soccer goalies. In: Ruiz-del-Solar, J., Chown, E., Plöger, P.G. (eds.) RoboCup 2010. LNCS (LNAI), vol. 6556, pp. 374-385. Springer, Heidelberg (2010)

12. Allgeuer, P., Schwarz, M., Pastrana, J., Schueller, S., Missura, M., Behnke, S.: A ROS-based software framework for the NimbRo-OP humanoid open platform. In: Proceedings of 8th Workshop on Humanoid Soccer Robots, IEEE Int. Conf. on Humanoid Robots, Atlanta, USA (2013)

13. Schwarz, M., Behnke, S.: Compliant robot behavior using servo actuator models identified by iterative learning control. In: 17th RoboCup Int. Symposium (2013)

14. Allgeuer, P., Behnke, S.: Hierarchical and state-based architectures for robot behavior planning and control. In: Proceedings of 8th Workshop on Humanoid Soccer Robots, IEEE Int. Conf. on Humanoid Robots, Atlanta, USA (2013)

15. Missura, M., Behnke, S.: Omnidirectional capture steps for bipedal walking. In: Proceedings of IEEE Int. Conf. on Humanoid Robots (Humanoids) (2013)

16. Kuo, A.D., Donelan, J.M., Ruina, A.: Energetic consequences of walking like an inverted pendulum: step-to-step transitions. Exercise and Sport Sciences Reviews 33(2), 88-97 (2005)

17. Missura, M., Behnke, S.: Dynaped demonstrates lateral capture steps, http://www.ais.uni-bonn.de/movies/DynapedLateralCaptureSteps .wmv 